\title{
RATIONAL ORBITS ON THREE-SYMMETRIC PRODUCTS OF ABELIAN VARIETIES
}

\author{
ALBERTO ALZATI AND GIAN PIETRO PIROLA
}

\begin{abstract}
Let $A$ be an $n$-dimensional Abelian variety, $n \geq 2$; let $\mathrm{CH}_{0}(A)$ be the group of zero-cycles of $A$, modulo rational equivalence; by regarding an effective, degree $k$, zero-cycle, as a point on $S^{k}(A)$ (the $k$-symmetric product of $A$ ), and by considering the associated rational equivalence class, we get a map $\gamma: S^{k}(A) \rightarrow \mathrm{CH}_{0}(A)$, whose fibres are called $\gamma$-orbits.

For any $n \geq 2$, in this paper we determine the maximal dimension of the $\gamma$-orbits when $k=2$ or 3 (it is, respectively, 1 and 2), and the maximal dimension of families of $\gamma$-orbits; moreover, for generic $A$, we get some refinements and in particular we show that if $\operatorname{dim}(A) \geq 4, S^{3}(A)$ does not contain any $\gamma$-orbit; note that it implies that a generic Abelian four-fold does not contain any trigonal curve. We also show that our bounds are sharp by some examples.

The used technique is the following: we have considered some special families of Abelian varieties: $A_{t}=E_{t} \times B \quad\left(E_{t}\right.$ is an elliptic curve with varying moduli) and we have constructed suitable projections between $S^{k}\left(A_{t}\right)$ and $S^{k}(B)$ which preserve the dimensions of the families of $\gamma$-orbits; then we have done induction on $n$. For $n=2$ the proof is based upon the papers of Mumford and Roitman on this topic.
\end{abstract}

\section{INTRODUCTION}

Let $X$ be a $d$-dimensional smooth algebraic variety; a cycle $Z$ of codimension $r$ in $X$ is defined to be an element of the free Abelian group $\mathbf{C}^{r}(X)$ generated by the irreducible subvarieties of codimension $r$ on $X$. We are interested in zero-cycles, i.e. when $r=d$. Two zero-cycles $Z_{1}$ and $Z_{2}$ of $X$ are rationally equivalent if there exists a cycle $Z$ on $X \times \mathbf{A}^{1}$, which intersects each fibre $X \times\{t\}$ in some points such that $Z_{1}$ and $Z_{2}$ are obtained respectively by intersecting $Z$ with the fibres $X \times\{0\}$ and $X \times\{1\}$. Note that this is in fact an equivalence relation and that the zero-cycles rationally equivalent to 0 (the zero of $\mathbf{C}^{d}(X)$ ) form a subgroup of $\mathbf{C}^{d}(X)$, (see $\left[\mathrm{H}, \mathrm{R}_{1}\right]$ ).

We denote by $\mathrm{CH}_{0}(X)$ the (Chow) group of zero-cycles on $X$, modulo rational equivalence. If $Z=\sum n_{i} P_{i}$ is a zero-cycle, where the $P_{i}$ are points of $X$, we define the degree of $Z$ to be $\sum n_{i}$. It is convenient to regard an effective

\footnotetext{
Received by the editors July 9, 1990 and, in revised form, December 10, 1990 and March 25, 1991.

1991 Mathematics Subject Classification. Primary 14C25; Secondary 14K99.

Key words and phrases. Abelian varieties, rational equivalence, zero-cycles.

Both authors are members of G.N.S.A.G.A. of the Italian C.N.R.

This research has been done within the framework of the national project "Geometria Algebrica". 
zero-cycle $Z=\sum n_{i} P_{i}$ i.e. one where all the $n_{i}>0$, as a point on the $k$ th symmetric product $S^{k}(X)$ of $X$, where $k=\operatorname{deg}(Z)$. Then by taking the associated rational equivalence class, we obtain a map $\gamma: S^{k}(X) \rightarrow \mathrm{CH}_{0}(X)$; the fibres of this map will be called $\gamma$-orbits; the irreducible, connected, components of a $\gamma$-orbit will be called $\gamma$-components, $(\gamma$-curves if they have dimension 1 , $\gamma$-surfaces if they have dimension 2 , etc.).

Now let $A$ be an Abelian variety, if we consider the Albanese morphism $\alpha_{k}: S^{k}(A) \rightarrow \operatorname{Alb}\left[S^{k}(A)\right]=A$ (i.e. $\left.\alpha_{k}\left(x_{1}, x_{2}, \ldots, x_{k}\right)=x_{1}+x_{2}+\cdots+x_{k}\right)$, we have that the fibres of $\alpha_{k}$ are all isomorphic and that every $\gamma$-orbit of $S^{k}(A)$ is contained in exactly one fibre of $\alpha_{k}$. Then, if we want to study the $\gamma$-orbits of $S^{k}(A)$, we have only to consider the $\gamma$-orbits contained in $K_{k}(A)=\operatorname{ker}\left(\alpha_{k}\right)$.

In [P] the author showed that for a generic Abelian variety $A$, with $\operatorname{dim}(A) \geq 3$, its Kummer variety, $K(A)$, does not contain any rational curve. By remarking that $K(A)$ is $K_{2}(A)$ in the previous notations, you can think that in $S^{2}(A)$ there are no one-dimensional $\gamma$-orbits, (where "dimension" means: maximal dimension of the $\gamma$-components of the $\gamma$-orbit, see $\S 3$ ). In fact, as Clemens pointed out, the technique used in $[\mathrm{P}]$ is related to the famous Mumford's paper [M] about the rational equivalence of zero cycles on a surface. So that, by those arguments, it is possible to show:

Theorem (1.1). Let $A$ be an Abelian variety, $\operatorname{dim}(A) \geq 2$, then

(a) $S^{2}(A)$ does not contain any two-dimensional $\gamma$-orbit;

(b) if $A$ is generic and $\operatorname{dim}(A) \geq 3, S^{2}(A)$ does not contain any onedimensional $\gamma$-orbit.

The proof of $(1.1)$ is essentially contained in $[\mathrm{P}]$ : you have only to change the words "rational curve" into " $\gamma$-curve", (see also (7.1)).

In this paper we study the $\gamma$-orbits of $S^{3}(A), \operatorname{dim}(A) \geq 2$, and we obtain the following results:

Theorem (1.2). Let $A$ be an Abelian variety, $\operatorname{dim}(A) \geq 2$, then

(a) in $S^{3}(A)$ there are no $d$-dimensional $\gamma$-orbits with $d \geq 3$;

(b) in $K_{3}(A)$ there are no one-dimensional families of two-dimensional $\gamma$ orbits;

(c) if $\operatorname{dim}(A)=2$, in $K_{3}(A)$ there are no three-dimensional families of one-dimensional $\gamma$-orbits.

Remark (1.3). If $\operatorname{dim}(A)=2$, in $S^{3}(A)$ there are some two-dimensional $\gamma$ orbits and some two-dimensional families of one-dimensional $\gamma$-orbits, see Examples (5.2) and (5.3); so that (1.2) is sharp.

Theorem (1.4). Let $A$ be a generic Abelian variety, $\operatorname{dim}(A) \geq 3$, then

(a) if $\operatorname{dim}(A)=3$, in $S^{3}(A)$ there are no two-dimensional $\gamma$-orbits;

(b) if $\operatorname{dim}(A)=3$, in $K_{3}(A)$ there are no two-dimensional families of onedimensional $\gamma$-orbits;

(c) if $\operatorname{dim}(A) \geq 4$, in $S^{3}(A)$ there are no one-dimensional $\gamma$-orbits.

The proof of (1.2), in $\S 5$, is based upon the results of Mumford and Roitman (see $\S 3$ ); but, to apply them, we have needed some linear algebra which we have condensed in $\S 4$. 
To prove (1.4) we have considered some special families of Abelian varieties of this type: $A_{t}=E_{t} \times B$ (where $E$ is usually an elliptic curve with varying moduli), and we have used the projections between $S^{3}\left(A_{t}\right)$ and $S^{3}(B)$ which preserve the dimension of the families of $\gamma$-orbits, then we have applied (1.2) to $S^{3}(B)$, (see $\left.\S 7\right)$.

Unfortunately we did not find an easy way to show that such projections do exist, not even when $A$ is isogenous to a product of elliptic curves. So we were forced to prove the lemmas in $\S 6$; actually some proof could be shortened by using the De Franchis-Severi theorem (for curves and for surfaces, see [D$\mathrm{M}]$ ), but we have avoided this theorem, firstly since it is not strictly necessary, secondly since we hope to generalize our results to $S^{k}(A), k \geq 4$.

Our theorems have the following corollary, which solves the problem put at the end of $[\mathrm{P}]$ :

Corollary (1.5). Let $A$ be a generic $g$-dimensional Abelian variety, $g \geq 4$. Then $A$ is not a quotient of a Jacobian of a trigonal curve, in other words $A$ does not contain trigonal curves.

Proof. Let $C$ be a trigonal curve such that there exists a surjective map

$$
f: J(C) \rightarrow A .
$$

By composing $f$ with the Abel-Jacobi map, we get a nontrivial map $C \rightarrow A$, hence we have a finite map: $S^{3}(C) \rightarrow S^{3}(A)$; as $C$ is trigonal we have another obvious map: $\mathbf{P}^{1} \rightarrow S^{3}(C) \rightarrow S^{3}(A)$; this gives rise to a rational curve in $S^{3}(A)$, but it is not possible by $(1.4)(\mathrm{c})$.

Remark (1.6). Obviously the Jacobian of a trigonal curve contains a trigonal curve: the curve itself; (1.5) shows that, among Abelian varieties, the Jacobians of genus 4 curves are special also under this point of view.

Acknowledgments. We wish to thank $\mathrm{H}$. Clemens who called all these problems to our attention and the referee for his suggestions.

\section{NOTATIONS AND CONVENTIONS}

\begin{tabular}{|c|c|}
\hline & direct sum of vector spaces, \\
\hline$\left\langle\mathbf{x}_{1}, \mathbf{x}_{2}, \ldots\right\rangle$ & C-vector space generated by $\mathbf{x}_{1}, \mathbf{x}_{2}, \ldots$ \\
\hline variety & by this term we mean a projective complex variety, \\
\hline$n$-fold & $n$-dimensional variety (not necessarily smooth), \\
\hline surface & two-fold \\
\hline curve & one-fold, \\
\hline generic & $\begin{array}{l}\text { by this word we mean: outside a countable union of proper } \\
\text { analytic subvarieties, }\end{array}$ \\
\hline$K_{V}$ & canonical divisor of the variety $V$ when it is smooth, \\
\hline$V \times V$ & Cartesian product of the variety $V$ with itself, \\
\hline$V^{k}$ & $k$-Cartesian product of the variety $V$ \\
\hline$S^{k}(V)$ & $k$-symmetric product of the variety $V$, \\
\hline & Siegel space of $n$-dimensional Abelian varieties. \\
\hline
\end{tabular}




\section{RATIONAL EQUIVALENCE OF ZERO-CYCLES}

In this paragraph we recall the results of Roitman and Mumford we need in the sequel.

Proposition (3.1) (see $\left[\mathrm{R}_{2}\right]$ ). Let $Z$ be a degree $k$ effective zero-cycle on $a$ smooth variety $X$, then the $\gamma$-orbit of $X$ containing $Z$ is a countable union of closed subsets of $S^{k}(X)$; such a set is usually called c-closed.

We can define the dimension of a $c$-closed set as the maximal dimension of its irreducible components. In this way it is possible to define the dimension of the image: $\gamma\left(S^{k}(X)\right) \subseteq \mathrm{CH}_{0}(X)$, even though it is not an algebraic variety, as

$$
d_{k}=\operatorname{dim}\left(S^{k}(X)\right)-\min \{\text { dimension of a fibre of } \gamma\} \text {. }
$$

We say that $\mathrm{CH}_{0}(X)$ is finite dimensional if the set of integers $d_{k}$ is bounded, otherwise we say that $\mathrm{CH}_{0}(X)$ is infinite dimensional.

In [M] Mumford proved that if $X$ is a surface with geometric genus $p_{g}>$ 0 , then $\mathrm{CH}_{0}(X)$ is infinite dimensional. In [ $\mathrm{R}_{2}$ ] Roitman gave the following generalization:

Theorem (3.2). Let $X$ be a smooth variety; then there are integers $d(X)$ and $j(X) \geq 0$, and an integer $k_{0}$, such that for all $k \geq k_{0}$ we have $d_{k}=k d(X)+$ $j(X)$. Moreover $d(X) \leq \operatorname{dim}(X)$, and $d(X)=0$ if and only if $\mathrm{CH}_{0}(X)$ is finite dimensional.

In $\left[R_{1}\right.$ and $R_{2}$ ] Roitman proved the following:

Theorem (3.3). Let $X$ be a smooth variety, suppose that, for some positive integer $q$, there exists a nonzero global $q$-form $\omega$ on $X$. Then $\omega$ induces a $q$-form $\omega_{k}$ on $S^{k}(X)$ whose restriction to any $\gamma$-component of $S^{k}(X)$ is zero. Hence $d(X) \geq q$.

We recall that the $q$-form $\omega_{k}$ quoted in (3.3) is defined as follows: we consider $X^{k}$ and for any $i=1,2, \ldots, k$ we consider the natural projection onto the $i$ th factor $p_{i}: X^{k} \rightarrow X$, now the $q$-form $\sum p_{i}^{*} \omega$ is well defined at the generic point of $S^{k}(X)$ because it is invariant under the action of the symmetric group; so we set $\omega_{k}=\sum p_{i}^{*} \omega$. In the same papers Roitman also shows the following:

Theorem (3.4). Let $f_{1}, f_{2}$ be two maps between a smooth variety $V$ and $S^{k}(X)$ such that $\forall v \in V \quad f_{1}(v)$ is rationally equivalent to $f_{2}(v)$; let $\omega$ be a q-form defined on $X$; then $f_{1}^{*}\left(\omega_{k}\right)=f_{2}^{*}\left(\omega_{k}\right)$.

The previous theorem allows us to prove this corollary.

Corollary (3.5). Let $V$ be a smooth $n$-dimensional variety; let $f: V \rightarrow S^{k}(X)$ be a map; suppose that there exists a map $p: V \rightarrow B$, where $B$ is an $n-$ $t$ dimensional variety, such that $\forall b \in B, f\left[p^{-1}(b)\right]$ is a t-dimensional $\gamma$ component of $S^{k}(X)$; let $\omega$ be a q-form defined on $X$. Then $f^{*} \omega_{k}=0$ if $q>n-t$.

Proof. We can always choose a suitable subvariety $W$ of $V$ such that $p_{\mid W}$ is finite over $B$; let $V^{\#}$ be $V \times_{B} W$ (fibre product). Let $p^{\#}: V^{\#} \rightarrow W$ and $\pi^{\#}: V^{\#} \rightarrow V$ the induced projections and $\sigma: W \rightarrow V^{\#}$ be the canonical section 
of $p^{\#}$; now we consider the maps $h, g: V^{\#} \rightarrow S^{k}(X)$ such that $h(v)=f\left[\pi^{\#}(v)\right]$ and $g(v)=h\left\{\sigma\left[p^{\#}(v)\right]\right\}$. Obviously $h(v)$ is rationally equivalent to $g(v)$ $\forall v \in V^{\#}$, and therefore, by (3.4), $h^{*} \omega_{k}=g^{*} \omega_{k}$. But $g^{*} \omega_{k}=\left(p^{\#}\right)^{*} \sigma^{*} h^{*} \omega_{k}$ and $\sigma^{*} h^{*} \omega_{k}=0$ if $q>n-t$, as $\pi^{*}$ is finite on $V, f^{*} \omega_{k}=0$.

\section{SOME LINEAR ALGEBRA}

Let $V$ be $\mathbf{C}^{2}$, and let $\{d z, d w\}$ be a basis for $V^{*}$. Let $L_{2}$ be the kernel of the map $\sigma: V \oplus V \oplus V \rightarrow V$ given by summation. Consider the following two-form on $L_{2}$ :

$$
\begin{aligned}
{\left[d z_{1}\right.} & \left.\wedge d w_{1}+d z_{2} \wedge d w_{2}+d z_{3} \wedge d w_{3}\right]_{\mid L_{2}} \\
& =\left[2 d z_{1} \wedge d w_{1}+2 d z_{2} \wedge d w_{2}+d z_{1} \wedge d w_{2}+d z_{2} \wedge d w_{1}\right]_{\mid L_{2}} \\
& =\left[d z_{1} \wedge d\left(2 w_{1}+w_{2}\right)+d\left(z_{1}+2 z_{2}\right) \wedge d w_{2}\right]_{\mid L_{2}} .
\end{aligned}
$$

As ( $)$ has maximal rank on $L_{2}$, we have that any locally isotropic subspace of $V \oplus V \oplus V$ for $\left({ }^{\wedge}\right)$, has dimension 2 at most. In fact there are such twodimensional maximal subspaces, for instance: $\left\{\left(\mathbf{v}, \rho \mathbf{v}, \rho^{2} \mathbf{v}\right), \mathbf{v} \in V, \rho \in \mathbf{C}\right.$ with $\left.1+\rho+\rho^{2}=0\right\}$.

Now let $W$ be $\mathbf{C}^{n}, n \geq 2$, and let $L_{n}$ be the kernel of the map $\sigma: W \oplus$ $W \oplus W \rightarrow W$ as before. Let $U$ be a linear subspace of $L_{n}$ such that for all projections $W \rightarrow V$, the induced map $L_{n} \rightarrow L_{2}$ sends $U$ into a totally isotropic subspace of $L_{2}$ for $\left({ }^{\wedge}\right)$. Then $\operatorname{dim}(U) \leq n$. In fact, for $n=2$ this is true, for $n \geq 3$ we can proceed by induction on $n$ : every projection $L_{n} \rightarrow L_{n-1}$ has kernel of dimension 2 , so that $\operatorname{dim}(U) \leq n+1$; moreover if $\operatorname{dim}(U)=n+1$, the kernel of every projection $L_{n} \rightarrow L_{n-1}$ would lie in $U$, and this is not possible.

Note that $\operatorname{dim}(U)=n$ is possible, for instance if $U=\left\{\left(\mathbf{w}, \rho \mathbf{w}, \rho^{2} \mathbf{w}\right)\right.$, $\mathbf{w} \in W, \rho \in \mathbf{C}$ with $\left.1+\rho+\rho^{2}=0\right\}$; we will see in (4.2) that it is the only possibility. Now we can prove the following:

Proposition (4.1). In the same notation as before, let $n=3$, let $\{d z, d w, d u\}$ be a basis for $W^{*}$; consider the following three-form:

$$
(\sim) \quad d z_{1} \wedge d w_{1} \wedge d u_{1}+d z_{2} \wedge d w_{2} \wedge d u_{2}+d z_{3} \wedge d w_{3} \wedge d u_{3}
$$

and suppose that $U$ is totally isotropic for $(\curvearrowright \sim)$. Then $\operatorname{dim}(U) \leq 2$.

Proof. By contradiction we suppose that $\operatorname{dim}(U)=3$, then by projecting $W$ to $V$ three times along the respective axes we see that:

$$
\begin{aligned}
U= & \left\langle\left(a_{1}, 0,0\right),\left(a_{2}, 0,0\right),\left(a_{3}, 0,0\right)\right\rangle+\left\langle\left(0, b_{1}, 0\right),\left(0, b_{2}, 0\right),\left(0, b_{3}, 0\right)\right\rangle \\
& +\left\langle\left(0,0, c_{1}\right),\left(0,0, c_{2}\right),\left(0,0, c_{3}\right)\right\rangle
\end{aligned}
$$

with: $\sum a_{i}=\sum b_{i}=\sum c_{i}=0$. So the vectors $\mathbf{a}=\left(a_{i}\right), \mathbf{b}=\left(b_{i}\right), \mathbf{c}=$ $\left(c_{i}\right)$ in $\mathbf{C}^{3}$ lie in the plane $P$ defined by the equation: $\sum x_{i}=0$. Since for all projections $W \rightarrow V$, the induced map $L_{n} \rightarrow L_{2}$ sends $U$ into a totally isotropic subspace of $L_{2}$ for $\left({ }^{\wedge}\right)$, we have: $\sum a_{i} b_{i}=\sum b_{i} c_{i}=\sum c_{i} a_{i}=0$.

Since the symmetric bilinear form on $\mathbf{C}^{3}$ which has the identity associated matrix (with respect to the standard base) has rank 2 on $P$, we conclude from the above equations that either $\mathbf{a}, \mathbf{b}$ or $\mathbf{c}$ is $\mathbf{0}$, (this is impossible as we have supposed that $\operatorname{dim}(U)=3$ ) or $\mathbf{a}, \mathbf{b}$ and $\mathbf{c}$ are all multiples of the same vector 
w with $\sum w_{i}=\sum\left(w_{i}\right)^{2}=0$. So that $\mathbf{w}$ can be taken to be some permutation of $\left(1, \rho, \rho^{2}\right)$. Hence we can write: $\mathbf{a}=A \mathbf{w}, \mathbf{b}=B \mathbf{w}$ and $\mathbf{c}=C \mathbf{w}$ for some nonzero complex numbers $A, B, C$. But if we apply ( ^) to these three vectors we have that the result is zero if and only if $A B C=0$, contradiction!

By (4.1) it is very easy to prove the following:

Proposition (4.2). In the previous notation: let $n \geq 4$. Then $U \subseteq\left\{\left(\mathbf{w}, \rho \mathbf{w}, \rho^{2} \mathbf{w}\right)\right.$, $\mathbf{w} \in W, \rho \in \mathbf{C}$ with $\left.1+\rho+\rho^{2}=0\right\}$ and if all projections of $W$ into $\mathbf{C}^{3}$ send $U$ into a totally isotropic subspace for $(\wedge)$, we have that $\operatorname{dim}(U) \leq 2$.

\section{Proof of (1.2) AND SOME EXAMPLES}

Let $A$ be an $n$-dimensional Abelian variety. Firstly we want to recall some useful facts about $S^{k}(A)$.

There is an action of the additive group $A$ on the variety $S^{k}(A)$ : for every $a \in A$ we have $T_{a}: S^{k}(A) \rightarrow S^{k}(A)$ such that for every $\left(x_{1}, x_{2}, \ldots, x_{k}\right) \in$ $S^{k}(A) \quad\left(T_{a}\left(x_{1}, x_{2}, \ldots, x_{k}\right)=\left(x_{1}+a, x_{2}+a, \ldots, x_{k}+a\right)\right.$. For every $a \in A$, $T_{a}$ is an isomorphism of $S^{k}(A)$ which we will call translation, by abuse of language.

If we consider the $n k$-dimensional Abelian variety $A^{k}$, we have that there is a (k!)-covering $p: A^{k} \rightarrow S^{k}(A)$ which is obviously ramified on the points $\left(x_{1}, x_{2}, \ldots, x_{k}\right)$ of $S^{k}(A)$ such that the $x_{i}$ are not all distinct. Moreover there is another obvious ( $k$ !)-covering $\pi: A^{k-1} \rightarrow K_{k}(A)\left(K_{k}(A)\right.$ is the kernel of the Albanese map, see $\S 1)$ such that $\pi\left(x_{1}, x_{2}, \ldots, x_{k-1}\right)=\left(x_{1}, x_{2}, \ldots, x_{k-1}\right.$, $\left.-x_{1}-x_{2} \cdots-x_{k-1}\right)$. Remark that any $d$-dimensional $\gamma$-component in $K_{k}(A)$ gives rise to a $d$-fold in $A^{k-1}$ via $\pi$.

Now we are able to prove (1.2); recall that, by the argument of $\S 1$, we have to study the $\gamma$-orbits contained in $K_{3}(A)$.

Proof of $(1.2)(a)$. Let $V$ be the dual of the Lie algebra of $A, \operatorname{dim}(V)=$ $\operatorname{dim}(A)=n$, and we recall that, for any Abelian variety $A, \forall q \geq 1, H^{q, 0}(A)=$ $\Lambda^{q}(V)$.

For any $\omega \in \Lambda^{q}(V), q \geq 2$, we consider the $q$-form $\phi(\omega)$ induced by $\omega$ on $S^{3}(A)$ in the following way: $\phi(\omega)=p_{*}\left(p_{1}^{*} \omega+p_{2}^{*} \omega+p_{3}^{*} \omega\right)$, where

$$
p: A^{3} \rightarrow S^{3}(A)
$$

and $p_{1}, p_{2}, p_{3}$ are the projections of $A \times A \times A$ on $A$.

The tangent space $U$ at every smooth point of any $\gamma$-orbit of $K_{3}(A)$ lies in $L_{n}$ (see $\left.\S 4\right) ; \phi(\omega)$ has to vanish on $U$, by Theorem (3.3), for any $\omega \in \Lambda^{q}(V)$, $q=2,3, \ldots, n$; this means that the assumptions of $(4.2)$ about the projections of $U$ are satisfied. Hence $\operatorname{dim}(U) \leq 2$; therefore every $\gamma$-orbit has dimension 2 at most.

Remark (5.1). The previous proof is based on the fact that all the forms belonging to $\phi\left(\Lambda^{q}(V)\right), q=2,3, \ldots, n$, have to vanish on the tangent spaces at the smooth points of any $\gamma$-component of $K_{3}(A)$. So we can say that, if a $d$-fold, contained in $K_{3}(A)$, has the same properties, then $d \leq 2$. 
Proof of (1.2)(b). If there would be such a family $\left\{S_{t}\right\}, t \in \mathbf{C}$, then in $K_{3}(A)$ we would get a three-fold $T$ which would be filled by two-dimensional $\gamma$ components. By using the same notations as in the proof of $(1.2)(\mathrm{a})$, we have that, by Corollary (3.5), the forms belonging to $\phi\left(\Lambda^{q}(V)\right), q=2,3, \ldots, n$, have to vanish on the tangent spaces at the smooth points of $T$, but this implies that $\operatorname{dim}(T) \leq 2$ by Remark (5.1): contradiction!

Proof of $(1.2)(\mathrm{c})$. If there would be a family $\left\{C_{\mathbf{r}}\right\}, \mathbf{r} \in \mathbf{C}^{3}$, of one-dimensional $\gamma$-orbits in $K_{3}(A)$ then $K_{3}(A)$ would be filled by one-dimensional $\gamma$-components and this is not possible by (3.2) and (3.3).

Now we prove, by some examples, that, when $\operatorname{dim}(A)=2$, the one-dimensional $\gamma$-orbits can span a three-fold in $S^{3}(A)$, and that there are two-dimensional $\gamma$-orbits.

Example (5.2). Let $A$ be an Abelian surface; let $C$ be a nonhyperelliptic genus 3 (smooth, irreducible) curve on $A$. If we consider the divisor $L$ supported by $C$, we get $L^{2}=4$ by the genus formula, and $h^{0}(L)=2$ by the Riemann-Roch and Kodaira vanishing theorems.

So $C$ moves in a pencil $\left\{C_{\mu}\right\}$ which has four base points: $A, B, C, D$. The adjunction formula yields: $K_{L}=L_{\mid L}$; so that $A+B+C+D$ is a canonical divisor on every curve $C_{\mu}$ of the pencil.

The canonical model $C_{\mu}^{\prime}$ of $C_{\mu}$ is a smooth plane quartic whose canonical series is cut by the lines, therefore the divisor of $C_{\mu}^{\prime}$ corresponding to $A+B+$ $C+D$ is cut on $C_{\mu}^{\prime}$ by a line.

Now we consider a point $P_{\mu}$ on $C_{\mu}$ and the linear series $g_{3}^{1}$ corresponding to the linear series $g_{3}^{1}$ cut on $C_{\mu}^{\prime}$ by the lines passing through the point corresponding to $P_{\mu}$. So that for every $\lambda \in \mathbf{P}^{1}$ we have a divisor: $P_{\mu}+Q_{\mu \lambda}+R_{\mu \lambda}+S_{\mu \lambda}$ on $C_{\mu}$. We choose an Abel map $\alpha_{\mu}: C_{\mu} \rightarrow J\left(C_{\mu}\right)$ such that $\alpha_{\mu}\left(P_{\mu}\right)=0$, hence, by Abel theorem, $\alpha_{\mu}\left(Q_{\mu \lambda}+R_{\mu \lambda}+S_{\mu \lambda}\right)=\tau_{P, \mu}$ is constant with respect to $\lambda$. The 3-ples: $\alpha_{\mu}\left(Q_{\mu \lambda}\right), \alpha_{\mu}\left(R_{\mu \lambda}\right), \alpha_{\mu}\left(S_{\mu \lambda}\right)$ in $J\left(C_{\mu}\right)$ gives rise to a rational curve in $S^{3}\left[J\left(C_{\mu}\right)\right]$ as $\lambda$ moves in $\mathbf{P}^{1}$.

We consider the following commutative diagram

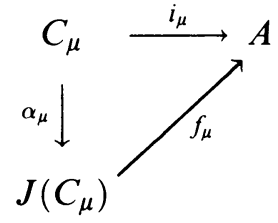

in which $i_{\mu}$ is the embedding of $C_{\mu}$ in $A$ and $f_{\mu}$ is the homomorphism between Abelian varieties induced by $\alpha_{\mu}$. By using $f_{\mu}$ we get a rational curve in $S^{3}(A)$; by translating this curve by $f_{\mu}\left(\tau_{P, \mu}\right)$ we get a rational curve $\gamma_{P, \mu}$ in $K_{3}(A)$.

Now we let $P$ vary on $C_{\mu}$ : for every point $P$ we get a curve $\gamma_{P, \mu}$ in $K_{3}(A)$; these curves are all distinct because the used linear series $g_{3}^{1}$ on $C_{\mu}^{\prime}$ are distinct. Now let $P$ vary on $C_{\mu}$ and let $\mu$ vary in $\mathbf{P}^{1}$ : for every couple $P, \mu$ we get a curve $\gamma_{P, \mu}$ in $K_{3}(A)$; these curves are all distinct because they are made by points lying on different curves $C_{\mu}$ of $A$. 
Obviously every curve $\gamma_{P, \mu}$ is contained in a $\gamma$-orbit of $K_{3}(A)$ and this example shows that in $K_{3}(A)$ there exist $\gamma$-orbits whose span is a three-fold.

Example (5.3). The previous example also shows that in $K_{3}(A)$ there exist some $\gamma$-orbits whose span is a surface. In fact for every curve $C_{\mu}$ of the previous example we can fix the point $A$, (one of the base points of the pencil $\left\{C_{\mu}\right\}$ ), and for every $\mu \in \mathbf{P}^{1}$ we get a rational curve $\gamma_{A, \mu}=\gamma_{\mu}$ in $K_{3}(A)$.

In this case, by recalling the construction of the linear series $g_{3}^{1}$, we have that for every $\mu \in \mathbf{P}^{1}$ there exists a $\lambda \in \mathbf{P}^{1}$ such that $Q_{\mu \lambda}=B, R_{\mu \lambda}=C$, $S_{\mu \lambda}=D$. Therefore: $\alpha_{\mu}(B+C+D)=\tau_{A, \mu}$ and $f_{\mu}\left[\alpha_{\mu}(B+C+D)\right]=f_{\mu}\left(\tau_{A, \mu}\right)=$ $i_{\mu}(B+C+D)$ is independent from $\mu$, hence the obtained curves in $S^{3}(A)$ belong to

$$
\left\{(x, y, z) \in S^{3}(A) \mid x+y+z=i_{\mu}(B+C+D)\right\}
$$

and all pass through the point: $\left(i_{\mu}(B), i_{\mu}(C), i_{\mu}(D)\right)$ in $S^{3}(A)$.

So that the translated curves $\gamma_{\mu}$ in $K_{3}(A)$ all intersect between them. Therefore the curves $\gamma_{\mu}$ span a rational surface in $K_{3}(A)$ which is contained in a $\gamma$-orbit.

\section{THE LEMMAS}

In this paragraph we prove some lemmas which will be useful in $\S 7$. We will need to study the projections of $d$-dimensional $\gamma$-components which are induced by natural projections between $K_{3}(V \times W)$ and $K_{3}(W)$, where $V$ and $W$ will be suitable Abelian varieties.

By the commutativity of the following diagram

$$
\begin{array}{cc}
(V \times W) \times(V \times W) & \longrightarrow W \times W \\
\downarrow \pi & \downarrow \pi \\
K_{3}(V \times W) & \longrightarrow K_{3}(W)
\end{array}
$$

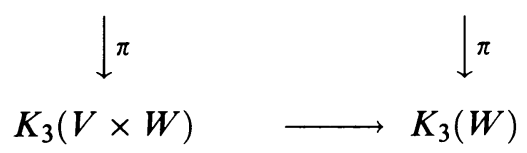

we have to study the natural projections $(V \times W) \times(V \times W) \rightarrow W \times W$, this is the aim of the following two lemmas.

Let $X$ be a smooth irreducible $d$-fold and let $A$ be an $n$-dimensional Abelian variety; let $\sigma: X \rightarrow A \times A$ be a map, birational onto its image, such that $\sigma(X)$ generates $A \times A$. Assume that $A$ is isogenous to $D \times D \times B$ where $D$ and $B$ are Abelian varieties of dimension $q$ and $(n-2 q)$ respectively. We fix two "dual" isogenies $D \times D \times B \rightarrow A \rightarrow D \times D \times B$ such that their composition is the multiplication by an integer; in this way we get a map $f \circ \sigma: X \rightarrow B \times B$ by composing the natural projection $f$ with $\sigma$; let $Y$ be $f[\sigma(X)]$; assume that (*) the natural projection $f: A \times A \rightarrow B \times B$ is such that
$Y=f[\sigma(X)]$ is a $d$-dimensional subvariety of $B \times B$.

Now let $\nu_{i}: D \rightarrow D \times D \rightarrow A$ be the composition of an embedding of $D$ in $D \times D$ with the previously chosen isogeny; we can suppose that $i$ varies in a countable set, in fact among all embeddings $D \rightarrow D \times D$ there are the following morphisms of algebraic groups: $\mathbf{d} \rightarrow(a \mathbf{d}, b \mathbf{d}$ ) (for any $\mathbf{d} \in D$ and for a fixed couple of coprime integers $a, b)$. We set $B_{i}=\left[(D \times D) / \nu_{i}(D)\right] \times B$ and let $X_{i}$ be the image of $X$ under the composition of the natural projection $A \times A \rightarrow B_{i} \times B_{i}$ with $\sigma$. 
In this situation we have the maps: $q_{i}^{*}: H^{1}\left(X_{i}, \mathbf{Q}\right) \rightarrow H^{1}(X, \mathbf{Q})$ and $\sigma^{*}: H^{1}(A \times A, \mathbf{Q}) \rightarrow H^{1}(X, \mathbf{Q})$; let $\Lambda_{i}$ be the image of $q_{i}^{*}$, then

Lemma (6.2). With the previous notations, there exists an index $i$ at least (hence an embedding of $D$ in $D \times D)$ such that $\Lambda_{i}$ contains the image of $\sigma^{*}$.

Proof. Note that this proof actually shows more, i.e. $\Lambda_{i}$ contains the image of $H^{1}(A \times A, \mathbf{Q})$ in $H^{1}(X, \mathbf{Q})$ save for a finite number of $i$.

For every $i$ we have a diagram of equidimensional $d$-folds

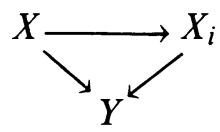

(the map $X_{i} \rightarrow Y$ is obtained by using the natural projection

$$
B_{i} \rightarrow B_{i} /\left[(D \times D) / \nu_{i}(D)\right]
$$

and by remarking that $B_{i} /\left[(D \times D) / \nu_{i}(D)\right]$ is isogenous to $\left.B\right)$. It follows that: $K[Y] \subset K\left[X_{i}\right] \subset K[X]$ so that there are only a finite number of birational models for the $X_{i}$. The maps in the following diagram are defined in the obvious way:

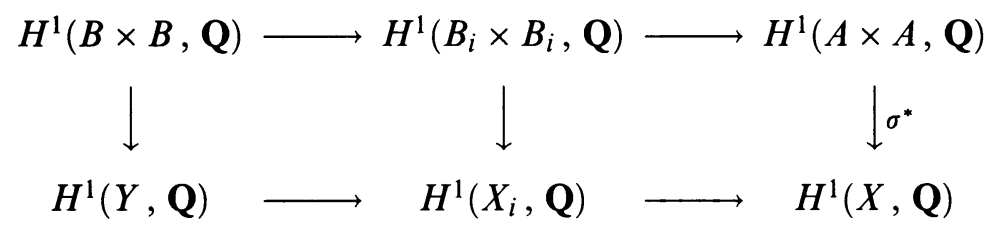

and we remark that, as $\sigma(X)$ generates $A \times A$ and the natural projection $A \times A \rightarrow B_{i} \times B_{i}$ is surjective, the map $H^{1}\left(B_{i} \times B_{i}, \mathbf{Q}\right) \rightarrow H^{1}(X, \mathbf{Q})$ is injective for any $i$. Now if we choose two distinct, transverse, embeddings of $D$ in $D \times D$ for which the corresponding fields $K\left[X_{i 1}\right]$ and $K\left[X_{i 2}\right]$, contained in $K[X]$, coincide, then we have that $H^{1}\left(X_{i 1}, \mathbf{Q}\right) \rightarrow H^{1}(X, \mathbf{Q})$ and $H^{1}\left(X_{i 2}, \mathbf{Q}\right) \rightarrow H^{1}(X, \mathbf{Q})$ are the same map; by the injectivity of $H^{1}\left(B_{i j} \times B_{i j}, \mathbf{Q}\right) \rightarrow H^{1}(X, \mathbf{Q}), j=1,2$, we have that $\Lambda_{i 1}=\Lambda_{i 2}$ must contain the span of the images of $H^{1}\left(B_{i 1} \times B_{i 1}, \mathbf{Q}\right)$ and $H^{1}\left(B_{i 2} \times B_{i 2}, \mathbf{Q}\right)$ in $H^{1}(X, \mathbf{Q})$, hence $\Lambda_{i 1}=\Lambda_{i 2}$ must contain the image of $H^{1}(A \times A, \mathbf{Q})$ in $H^{1}(X, \mathbf{Q})$.

Lemma (6.3). With the same assumptions as in (6.2), we get the same thesis if we consider $F^{1} H^{1}(-, C)$, (in the sense of mixed Hodge structures, see [G]), instead of $H^{1}(-, \mathbf{Q})$.

Remark (6.4). Note that, if $\operatorname{dim}(X)=1,(*)$ is always satisfied, (save, obviously, when $A=E \times E \times B, E$ elliptic curve, and $X=E$ ).

Now let $\Delta$ be an analytic scheme $(0 \in \Delta)$, and $h: \mathbf{A} \rightarrow \Delta$ a proper fibration such that $h^{-1}(t), t \in \Delta$, is an Abelian variety isogenous to $\mathbf{D}_{t} \times \mathbf{B}, \mathbf{B}$ fixed, $\left(h^{-1}(0)\right.$ isogenous to $\left.\mathbf{D}_{0} \times \mathbf{B}\right)$.

The infinitesimal variation of the Hodge structures induces the following map $\phi: H^{1,0}\left(\mathbf{D}_{0}\right) \rightarrow \operatorname{Hom}\left(T_{\Delta}(0), H^{0,1}\left(\mathbf{D}_{0}\right)\right)$, such that for any $\mu \in H^{1,0}\left(\mathbf{D}_{0}\right)$ and for any $\mathbf{t} \in T_{\Delta}(0), \phi(\mu)(\mathbf{t})$ is the derivative of $\mu$ along $\mathbf{t}$. We have the following: 
Lemma (6.5). With the previous assumptions, consider the commutative diagram

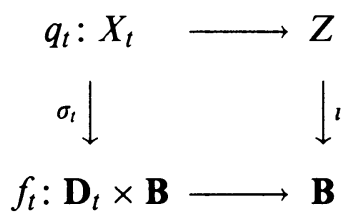

where $X_{t}$ are varieties parametrized by $t, \sigma_{t}$ are maps birational onto their images, $\sigma_{t}\left(X_{t}\right)$ generates $\mathbf{D}_{t} \times \mathbf{B}$ for any $t, f_{t}$ is the natural projection, $q_{t}$ is induced by $f_{t}, l$ is an inclusion and $Z$ is fixed. Assume that $\phi$ is injective; then

$$
\sigma_{0}^{*}\left[H^{1,0}\left(\mathbf{D}_{0}\right)\right] \cap q_{0}^{*} F^{1} H^{1}(Z)=0 \in F^{1} H^{1}\left(X_{0}\right) .
$$

Proof. If $\mu$ belongs to that intersection, $\phi(\mu)=0$ as $F^{1} H^{1}(Z)$ is independent from $t$; as $\phi$ is injective we have $\mu=0$.

Now let $\Delta$ be an open set of $\mathscr{H}_{n},(0 \in \Delta)$, we will call a " $(\Delta, m, G)$ situation" (for $\mathscr{H}_{n}$ ) the following data:

(i) a bundle of Abelian varieties over $\Delta: \mathbf{A} \times{ }_{\Delta} \mathbf{A} \times{ }_{\Delta} \cdots \times G$ ( $m$ times) where $\mathbf{A}$ is the tautological Abelian bundle over $\Delta$ and $G$ is a constant Abelian variety; (by abuse of notation we write $G=G \times \Delta$ and $\mathbf{A}^{m} \times G=\mathbf{A} \times{ }_{\Delta} \mathbf{A} \times{ }_{\Delta} \cdots \times G$ ( $m$ times));

(ii) a family of $d$-dimensional varieties $k: \mathbf{X} \rightarrow \Delta$ over $\Delta$;

(iii) a morphism of $\Delta$ families $\sigma: \mathbf{X} \rightarrow \mathbf{A}^{m} \times G$, i.e. a commutative diagram as follows:

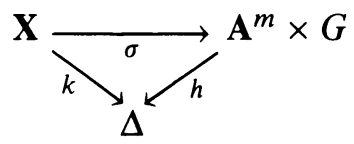

(we set $X_{t}=k^{-1}(t)$ and $\mathbf{h}^{-1}(t)=\left(A_{t}\right)^{m} \times G$ for any $\left.t \in \Delta\right)$;

(iv) the assumption that the image $\sigma_{t}\left(X_{t}\right)$ generates $\left(A_{t}\right)^{m} \times G$ as a group, for any $t \in \Delta$.

We remark that, if conditions (i), (ii), (iii) are satisfied, the bundle of Abelian varieties generated by the images $\sigma_{t}\left(X_{t}\right)$ must be isomorphic to $\mathbf{A}^{m^{\prime}} \times G^{\prime}$ where $m^{\prime} \leq m$ and $G^{\prime}$ is an Abelian subvariety of $G$; so that, by changing the bundle, we always get a $\left(\Delta, m^{\prime}, G^{\prime}\right)$-situation. With the above warning we can say that to have a $(\Delta, m, G)$-situation is equivalent to have a $d$-dimensional variety in $A^{m} \times G$ where $A$ is generic in $\Delta$; (i.e. for any $t \in \Delta$ we have a $d$-fold $X_{t}$ in $\left.\left(A_{t}\right)^{m} \times G\right)$. Actually we usually will consider only the case: $m=2, G=0$, (hence $\mathbf{h}=h \times{ }_{\Delta} h$ ); for the sake of simplicity, from now on, this case will be simply called " $\Delta$-situation."

Lemma (6.6). We suppose to be in a $\Delta$-situation; we choose $A$ isogenous to $D \times D \times B$, (as in Lemma (6.2)), and for any linear embedding $\nu_{i}: D \rightarrow D \times D$ we fix an isogeny between $A$ and $\nu_{i}(D) \times\left[(D \times D) / \nu_{i}(D)\right] \times B$.

Let $\Delta_{i}=\left\{t \in \Delta \mid\right.$ the fibre of $h \times_{\Delta} h$ is $A_{t} \times A_{t}$ where $A_{t}$ is isogenous to $\left.\nu_{i}(D) \times D_{t} \times B, D_{t} \in \mathscr{H}_{q}\right\} ;$ let $A_{0}$ be isogenous to $A$ by the isogeny induced by the previously fixed one. This defines an embedding $\nu_{i}^{*}: \mathscr{H}_{q} \rightarrow \mathscr{H}_{n}$, such that $\Delta_{i}=\Delta \cap\left[\nu_{i}^{*}\left(\mathscr{H}_{q}\right)\right] ;$ we set $B_{i}=\nu_{i}(D) \times B$.

For any $t \in \Delta_{i}$, let $f_{i, t}: A_{t} \times A_{t} \rightarrow B_{i} \times B_{i}$ be the natural projection; if we assume (*) for the natural projection $f_{i, 0}: A \times A \rightarrow B \times B$ and $\sigma_{0}\left(X_{0}\right)$, we have 
that, save a finite number of $i$ at most, $f_{i, t}\left[\sigma_{t}\left(X_{t}\right)\right]$ is not a fixed subvariety of $B_{i} \times B_{i}$.

Proof. We proceed by contradiction: if (6.6) is false, then for any $i, f_{i, t}\left[\sigma_{t}\left(X_{t}\right)\right]$ is a fixed $d$-fold $X_{i}$ in $B_{i}$ for any $t$, and $X_{i}$ generates $B_{i}$. Then we have the following commutative diagram:

$$
\begin{array}{ccc}
q_{i, 0}: X_{0} & \longrightarrow & X_{i} \\
\downarrow^{\sigma_{0}} & & \downarrow^{l_{i}} \\
f_{i, 0}:(D \times D \times B)^{2} & \longrightarrow & B_{i} \times B_{i} .
\end{array}
$$

Note that we can apply Lemma (6.5) because we are in a $\Delta$-situation, so we have that $\sigma_{0}^{*}\left[H^{1,0}\left(D^{4}\right)\right] \cap\left(q_{i, 0}\right)^{*} F^{1} H^{1}\left(X_{i}\right)=0 \in F^{1} H^{1}\left(X_{0}\right)$ but, by Lemma (6.3), $\left(q_{i, 0}\right)^{*} F^{1} H^{1}\left(X_{i}\right)$ contains $\sigma_{0}^{*}\left[H^{1,0}\left(D^{4}\right)\right]$ except for a finite number of $i$, contradiction!

Lemma (6.7). We are supposed to be in a $\Delta$-situation; but now we choose $A$ isogenous to $D^{m} \times B$, and we consider the countable set of the linear embeddings $\nu_{i}: D^{p} \rightarrow D^{m} \quad\left(p \leq m\right.$, positive integers, $\left.D \in \mathscr{H}_{q}, B \in \mathscr{H}_{n-m q}\right)$. For any embedding $\nu_{i}$ we fix an isogeny between $A$ and $\nu_{i}\left(D^{p}\right) \times\left[D^{m} / \nu_{i}\left(D^{p}\right)\right] \times B$; let $\Delta_{i}=\left\{t \in \Delta \mid\right.$ the fibre of $h \times{ }_{\Delta} h$ is $A_{t} \times A_{t}$ where $A_{t}$ is isogenous to $\left.F_{t} \times\left[D^{m} / \nu_{i}\left(D^{p}\right)\right] \times B, F_{t} \in \mathscr{H}_{p q}\right\}, A_{0}$ is isogenous to $A$ as in the previous cases. This defines an embedding $\nu_{i}^{*}: \mathscr{H}_{p q} \rightarrow \mathscr{H}_{n}$ such that: $\Delta_{i}=\Delta \cap\left[\nu_{i}^{*}\left(\mathscr{H}_{p q}\right)\right]$; we set: $B_{i}=\left[D^{m} / \nu_{i}\left(D^{p}\right)\right] \times B$.

For any $t \in \Delta_{i}$, let $f_{i, t}: A_{t} \times A_{t} \rightarrow B_{i} \times B_{i}$ be the natural projection; if we assume $(*)$ for the natural projection $f_{i, 0}: A \times A \rightarrow B \times B$ and $\sigma_{0}\left(X_{0}\right)$, we have that, save a finite number of $i$ at most, $f_{i, t}\left[\sigma_{t}\left(X_{t}\right)\right]$ is not a fixed subvariety of $B_{i}$.

Proof. See the proof of (6.6).

To apply the above lemmas we need condition $(*)$; this is a crucial point: it allows us to avoid the use of the De Franchis-Severi theorem. When $X$ is of general type and $d=1$ or 2 , this theorem would assure the existence of a finite number of subfields $K\left[X_{i}\right]$ of $K[X]$ (see the proof of Lemma (6.2)), without the assumption that $f$ is generically finite, i.e., roughly speaking, without fixing a shield $Y=f[\sigma(X)]$.

We use the following remark: consider diagram (6.1): our natural projections between $(V \times W) \times(V \times W)$ and $W \times W$ are induced by natural projections between $K_{3}(V \times W)$ and $K_{3}(W)$, so that to verify $(*)$ it suffices to verify the corresponding statement for projections between $K_{3}(V \times W)$ and $K_{3}(W)$, and vice versa. This explains the statements of the following other lemmas.

Lemma (6.8). Let $S$ be a $\gamma$-surface in $K_{3}(E \times E)$ where $E$ is a generic elliptic curve (in the sense of moduli); let $S^{\prime}$ be the pullback of $S$ in $E^{2} \times E^{2}$; let $E_{p q}$ be a fixed embedding of $E \times E$ in $E^{2} \times E^{2}$ such that $E_{p q}=\{p x, q x, p y, q y\}$ where $(x, y) \in E \times E$ and $p, q$ are coprime integers. Then there exist infinitely many couples $(p, q)$ such that $E_{p q}$ intersects $S^{\prime}$ properly. In these cases the natural projection $E^{2} \times E^{2} \rightarrow\left(E^{2} \times E^{2}\right) / E_{p q}$ is generically finite on $S^{\prime}$ (and the induced map $K_{3}(E \times E) \rightarrow K_{3}[(E \times E) /\{p x, q y\}]$ is generically finite on $\left.S\right)$. 
Proof. We will prove that there exists a couple $(p, q)$ at least, such that $E_{p q}$ intersects $S^{\prime}$ properly, but, in fact, our proof will also show that the intersection is proper save for a finite number of couples.

We proceed by contradiction; we recall that if two surfaces in $E^{4}$ does not intersect properly then, for every generic point of the first surface, there passes a translate of the second one which intersects the former one along a curve. In fact the intersection cycle of two surfaces in $E^{4}$ depends only on their homology class, and the homology class is invariant under translations.

We fix a generic point $P$ of $S^{\prime}$, if every $E_{p q}$ does not intersect $S^{\prime}$ properly then, $\forall p, q$, there exists a translate of $E_{p q}$ passing through $P$ and cutting $S^{\prime}$ along a curve; hence, by looking at the tangent spaces, we have that in the Lie algebra of $E^{4}$ there are: a vector space generated by $(p, q, 0,0)$ and $(0,0, p, q), \forall p, q$, and the vector space $\left\langle\left(a_{1}, a_{2}, a_{3}, a_{4}\right),\left(b_{1}, b_{2}, b_{3}, b_{4}\right)\right\rangle$ (corresponding to the tangent space to $S^{\prime}$ at $P$ ), such that the matrix:

$$
\begin{array}{llll}
p & 0 & a_{1} & b_{1} \\
q & 0 & a_{2} & b_{2} \\
0 & p & a_{3} & b_{3} \\
0 & q & a_{4} & b_{4}
\end{array}
$$

is always singular. Now we show that, for generic $E$, this situation is not possible.

As $\left(a_{1}, a_{2}, a_{3}, a_{4}\right)$ and $\left(b_{1}, b_{2}, b_{3}, b_{4}\right)$ are independent, it is possible to choose a base for the Lie algebra such that: $a_{1}=b_{2}=1, b_{1}=a_{2}=0$; otherwise is not possible that the previous matrix is singular $\forall p, q$. Now it is easy to see that it is possible only if $b_{3}=a_{4}=0$ and $b_{4}=a_{3}=\rho$, with $\rho \in \mathbf{C}$. As $S^{\prime}$ is the pullback in $E \times E \times E \times E$ of a $\gamma$-component $S$ in $K_{3}(E \times E)$ which is not contained in the branching locus of $\pi$, the skew symmetric two-form ( $\curvearrowright$ ) considered in $\S 4$ has to vanish on the tangent space at the generic point $P$ of $S^{\prime}$ by (3.3), hence: $1+\rho+\rho^{2}=0$ and $\rho$ is a constant, independent from $P$.

This means that the only surfaces in $E^{4}$ which does not intersect properly $E_{p q} \forall p, q$, are, up to translations, those Abelian surfaces $S^{\prime}$ which are the embeddings of $E \times E$ in $E^{4}$ such that $S^{\prime}=\{x, y, \rho x, \rho y\}$ where $(x, y) \in$ $E \times E$ and $\rho \in \mathbf{C}$ with $1+\rho+\rho^{2}=0$; but this implies that $E$ has an endomorphism: $x \rightarrow \rho x \forall x \in E$, with $1+\rho+\rho^{2}=0$, and this is not possible for generic $E$.

Lemma (6.9). Let $S$ be a $\gamma$-surface in $K_{3}(E \times E \times E)$ where $E$ is a generic elliptic curve; let $S^{\prime}$ be the pullback of $S$ in $E^{3} \times E^{3}$; let $E\left(p, q, r, p^{\prime}, q^{\prime}, r^{\prime}\right)$ be a fixed embedding of $E^{2} \times E^{2}$ in $E^{3} \times E^{3}$ such that $E\left(p, q, r, p^{\prime}, q^{\prime}, r^{\prime}\right)=\{p x+$ $\left.p^{\prime} y, q x+q^{\prime} y, r x+r^{\prime} y, p z+p^{\prime} w, q z+q^{\prime} w, r z+r^{\prime} w\right\}$ where $(x, y, z, w) \in$ $E^{2} \times E^{2}$, and $(p, q, r),\left(p^{\prime}, q^{\prime}, r^{\prime}\right)$ are triple of coprime integers, and such that the following matrix has rank 2 :

$$
\begin{array}{lll}
p & q & r \\
p^{\prime} & q^{\prime} & r^{\prime}
\end{array}
$$

Then there exist infinitely many choices $\left(p, q, r, p^{\prime}, q^{\prime}, r^{\prime}\right)$ such that $E\left(p, q, r, p^{\prime}, q^{\prime}, r^{\prime}\right)$ intersects $S^{\prime}$ properly. In these cases the natural projection

$$
E^{3} \times E^{3} \rightarrow\left(E^{3} \times E^{3}\right) / E\left(p, q, r, p^{\prime}, q^{\prime}, r^{\prime}\right)
$$


is generically finite on $S^{\prime}$,

$$
\left(K_{3}(E \times E \times E) \rightarrow K_{3}\left[(E \times E \times E) /\left\{p x+p^{\prime} y, q x+q^{\prime} y, r x+r^{\prime} y\right\}\right]\right.
$$

is generically finite on $S$ ).

Proof. We can proceed as in the proof of Lemma (6.8).

Lemma (6.10). Let $E$ be a generic elliptic curve and let $T$ be a three-fold in $K_{3}\left(E^{3}\right)$ which is filled by a two-dimensional family of $\gamma$-curves; let $T^{\prime}$ be the pullback of $T$ in $E^{3} \times E^{3}$; let $E_{p q r}$ be a fixed embedding of $E \times E$ in $E^{3} \times E^{3}$ such that $E_{p q r}=\{p x, q x, r x, p y, q y, r y\}$ where $(x, y) \in E \times E$ and $p, q, r$ are coprime integers. Then there exist infinitely many triples $(p, q, r)$ such that $E_{p q r}$ does not intersect $T^{\prime}$ or intersects $T^{\prime}$ in a finite number of points. In these cases the natural projection $E^{3} \times E^{3} \rightarrow\left(E^{3} \times E^{3}\right) / E_{p q r}$ is generically finite on $T^{\prime}$ (and the induced map $K_{3}\left(E^{3}\right) \rightarrow K_{3}\left[E^{3} /\{p x, q x, r x\}\right]$ is generically finite on $T)$.

Proof. By arguing as in Lemma (6.8) we get that the only three-folds in $E^{3} \times E^{3}$ which does not intersect properly $E_{p q r} \forall p, q, r$ are, up to translations, those Abelian three-folds $T^{\prime}$ which are the embeddings of $E \times E \times E$ in $E^{3} \times E^{3}$ such that $T^{\prime}=\{x, y, z, s x, s y, s z\}$ where $(x, y, z) \in E \times E \times E$ and $s \in \mathbf{C}$ with $s(s+1)=0$.

This would imply that, in $K_{3}\left(E^{3}\right), T$ would be given by the unordered triples: $\{P, s P,-(s+1) P\}$, where $s=0$ or $s=-1$ and $P \in E^{3}$; in any case we could define an embedding $\lambda: T \rightarrow K_{2}\left(E^{3}\right)$ such that

$$
\lambda(\{P, s P,-(s+1) P\})=\{P,-P\} ;
$$

$\lambda(T)$ would be a three-fold filled out by $\gamma$-curves; but this is not possible by (1.1)(b): recall that $E$ is generic and the locus of nonsimple Abelian three-folds is dense in $\mathscr{H}_{3}$.

\section{Proof of (1.4)}

For the sake of simplicity, in every $\Delta$-situation considered in $\S 7$ we will identify $X_{t}$ with $\sigma_{t}\left(X_{t}\right)$.

Proof of $(1.4)(a)$. We proceed by contradiction: we assume that for any threedimensional Abelian variety $A, S^{3}(A)$, and therefore $K_{3}(A)$, contains a $\gamma$ surface; by their pullback via $\pi$ we have a surface in any $A^{2}$, so we are in a $\Delta$-situation. Then we can construct a fibration $h \times_{\Delta} h: \mathbf{A} \times_{\Delta} \mathbf{A} \rightarrow \Delta \subset \mathscr{H}_{3}$ as in $\S 6$. We want to apply Lemma (6.6) with $D=B=E, E$ generic elliptic curve. To have $(*)$ we use Lemma (6.9): we can fix an Abelian variety $A$ isogenous to $E \times E \times E$, such that, when we project the $\gamma$-surface $X$ contained in $K_{3}(E \times E \times E)$ into $K_{3}(E)$ (the last factor), by the natural projection, we obtain another $\gamma$-surface $Y$. This means that the natural projection $f: A \times A \rightarrow B \times B$ satisfies $(*)$.

Now let $E_{p q}$ be the image in $E \times E$ of the embedding $\nu_{p q}$ of $E$ such that $\nu_{p q}(x)=(p x, q x) \quad \forall x \in E,(p, q)$ is a couple of coprime integers. We fix an isogeny between $A$ and $E_{p q} \times B_{p q}$ where $B_{p q}$ is $\left[(E \times E) / E_{p q}\right] \times E$. Let $\Delta_{p q}=\Delta \cap\left[\nu_{p q}^{*}\left(\mathscr{H}_{1}\right)\right]$ the open subset of $\Delta$ such that the fibre over $t \in \Delta_{p q}$ is 
$A_{t} \times A_{t}$ where $A_{t}$ is isogenous to $E_{t} \times B_{p q}$ ( $A_{0}$ isogenous to $A$ by the previously fixed isogeny) and $E_{t}$ is an elliptic curve whose moduli depend on $t$.

Let $\varphi_{t}$ be the natural projection between $K_{3}\left(A_{t}\right)$ and $K_{3}\left(B_{p q}\right)$, by our assumption there is a $\gamma$-surface $X_{t}$ in every $A_{t}$ and $X_{0}=X$. For small $t$, we can assume that $Y_{t}=\varphi_{t}\left(X_{t}\right)$ is a $\gamma$-surface of $K_{3}\left(B_{p q}\right)$; in fact $Y_{0}=\varphi_{0}\left(X_{0}\right)=$ $\varphi_{0}(X)$ is a surface in $K_{3}\left(B_{p q}\right)$ because $X$ projects into a surface in $K_{3}(E)$.

By Lemma (6.6), we can choose $(p, q)$ such that $\left\{Y_{t}\right\}$ is a one-dimensional family of $\gamma$-surfaces of $K_{3}\left(B_{p q}\right)$ (i.e. the union of the $Y_{t}$ span a three-fold in $\left.K_{3}\left(B_{p q}\right)\right)$; but $\operatorname{dim}\left(B_{p q}\right)=2$ and this is a contradiction with (1.2)(b).

Remark (7.1). Here we want to give a short outline of the proof of $(1.1)(\mathrm{b})$ when $\operatorname{dim}(A)=3$. Firstly we need $(1.1)(\mathrm{a})$ for dimension 2 : this is just an application of (3.2) and (3.3): if (1.1)(a) were false, for the generic point of $S^{2}(A)$ would pass a positive dimensional $\gamma$-orbit, but then $d_{2}$ would be strictly less than 4 .

Now we proceed by contradiction: we assume that for the generic Abelian three-fold $A, S^{2}(A)$, and therefore $K_{2}(A)$ (which is the Kummer variety $K(A)$ of $A$ ), contains a $\gamma$-curve. By their pullback via $\pi$ we get a curve in any $A$; by using these we can build a family of curves that gives rise to a $\Delta$-situation. By arguing as in the proof of $(1.4)($ a) we can choose a suitable projection from $K_{2}\left(A_{t}\right)=K\left(A_{t}\right)$ onto $K(E \times E)$, where $A_{t}$ is isogenous to $E_{t} \times E \times E, E$ generic elliptic curve, in such a way that the images of our curves cover $K(E \times E)$. Since the image of a $\gamma$-orbit is a $\gamma$-orbit, we get a contradiction with (1.1)(a).

Proof of $(1.4)(\mathrm{b})$. We proceed by contradiction: we assume that for any threedimensional Abelian variety $S^{3}(A)$, and therefore $K_{3}(A)$ contains a three-fold filled by $\gamma$-curves: by their pull-back via $\pi$ we have a three-fold in any $A^{2}$. So we are in a $\Delta$-situation and we can construct a fibration $h \times_{\Delta} h: \mathbf{A} \times{ }_{\Delta} \mathbf{A} \rightarrow \Delta \subset \mathscr{H}_{3}$ as in $\S 6$. Pay attention: now we proceed in a very similar way to the proof of $(1.4)(a)$, but we cannot use Lemma (6.6) in that manner.

We fix an Abelian variety $A$ isogenous to $E \times E \times E, E$ generic elliptic curve. Let $E_{p q r}$ be the image in $E \times E \times E$ of the embedding $\nu_{p q r}$ of $E$ such that $\nu_{p q r}(x)=(p x, q x, r x) \forall x \in E,(p, q, r)$ is a triple of coprime integers; let $F_{p q r}$ be $(E \times E \times E) / E_{p q r}$, we fix an isogeny between $A$ and $E_{p q r} \times F_{p q r}$.

By Lemma (6.10) we can assume that, when we project the three-fold $T$, filled by $\gamma$-curves, contained in $K_{3}(E \times E \times E)$, into $K_{3}\left(F_{p q r}\right)$, by the natural projection, we obtain another three-fold $T^{\#}$ with the same property.

Let $\Delta_{p q r}=\Delta \cap\left[\nu_{p q r}^{*}\left(\mathscr{H}_{1}\right)\right]$ the open subset of $\Delta$ such that the fibre over $t \in \Delta_{p q r}$ is $A_{t} \times A_{t}$ where $A_{t}$ is isogenous to $E_{t} \times F_{p q r}\left(A_{0}\right.$ isogenous to $\left.A\right)$ and $E_{t}$ is an elliptic curve whose moduli depend on $t$.

Let $\varphi_{t}$ be the natural projection between $K_{3}\left(A_{t}\right)$ and $K_{3}\left(F_{p q r}\right)$, by our assumption there is a three-fold $T_{t}$, filled by $\gamma$-curves, in every $A_{t}$ and $T_{0}=T$. Moreover $\varphi_{0}\left(T_{0}\right)=\varphi_{0}(T)=T \#$ is a three-fold in $K_{3}\left(F_{p q r}\right)$ by the previous remarks. Therefore, by choosing a smaller disk, we can assume that $T_{t}^{\#}=\varphi_{t}\left(T_{t}\right)$ is three-fold in $K_{3}\left(F_{p q r}\right)$.

We can use Lemma (6.6) (and Remark (6.4)), to assure that there exist triples $(p, q, r)$ (for instance with $r=0$ ) such that every one-dimensional family $\left\{C_{t}\right\}$ of $\gamma$-curves of $K_{3}\left(A_{t}\right)$ projects into another similar family of $K_{3}\left(F_{p q r}\right)$. We choose one of these triples. 
Now we consider two cases: if $T_{t}^{\#}$ is a variable three-fold in $K_{3}\left(F_{p q r}\right)$, by the previous condition, we would get a three-dimensional family of $\gamma$-curves in $K_{3}\left(F_{p q r}\right)$, but $\operatorname{dim}\left(F_{p q r}\right)=2$ and this is forbidden by $(1.2)(\mathrm{c})$.

If $T_{t}^{\#}=T_{0}^{\#}$ is a fixed three-fold in $K_{3}\left(F_{p q r}\right)$ then, by the previous condition, infinitely many $\gamma$-components pass through any point of $T_{0}^{\#}$, hence we would have a one-dimensional family of $\gamma$-surfaces in $K_{3}\left(F_{p q r}\right)$ at least, and this is not possible by $(1.2)(b)$.

Proof of $(1.4)(\mathrm{c})$. Firstly we assume that $\operatorname{dim}(A)=4$ and we proceed by contradiction: we assume that for any four-dimensional Abelian variety $A, S^{3}(A)$, and therefore $K_{3}(A)$ contains a $\gamma$-curve; by their pullback via $\pi$ we have a curve in any $A^{2}$. So we have a $\Delta$-situation and then we can construct a fibration $h \times_{\Delta} h: \mathbf{A} \times{ }_{\Delta} \mathbf{A} \rightarrow \Delta \subset \mathscr{H}_{4}$ as in $\S 6$.

We want to use Lemma (6.7) with $D=B=E, E$ generic elliptic curve, $p=2, m=3$; note that $(*)$ is satisfied by Remark (6.4).

We fix an Abelian variety $A$ isogenous to $E \times E \times E \times E$. For any embedding $\nu_{i}: E^{2} \rightarrow E^{3}$ let $\Delta_{i}=\Delta \cap\left[\nu_{i}^{*}\left(\mathscr{H}_{2}\right)\right]$ the open subset of $\Delta$ such that the fibre over $\mathbf{r} \in \Delta_{i}$ is $A_{\mathbf{r}} \times A_{\mathbf{r}}$ where $A_{\mathbf{r}}$ is isogenous to $D_{\mathbf{r}} \times\left[E^{3} / \nu_{i}\left(E^{2}\right)\right] \times E\left(A_{0}\right.$ isogenous to $A$ as usual), $D_{\mathrm{r}}$ is an Abelian surface and $D_{0}$ is isogenous to $E \times E$; in this case we set: $B_{i}=\left[E^{3} / \nu_{i}\left(E^{2}\right)\right] \times E$. By our assumptions there exists a one-dimensional $\gamma$-component $C_{\mathbf{r}}$ in any $K_{3}\left(A_{\mathbf{r}}\right)$.

Now we use Lemma (6.7) and we have that, for all $i$ except a finite number, when we project $K_{3}\left(A_{\mathrm{r}}\right)$ into $K_{3}\left(B_{i}\right)$, by the natural projection, we get that every one-dimensional family of $\gamma$-curves projects into a one-dimensional family of $\gamma$-curves.

So we get a three-dimensional family of $\gamma$-curves in $K_{3}\left(B_{i}\right)$; they cannot cover all $K_{3}\left(B_{i}\right)$ by Theorem (3.2) (recall that $B_{i}$ is isogenous to $E^{2}$ ); they cannot cover a three-fold, otherwise this three-fold would be filled by $\gamma$-surfaces and this is not possible by (1.2)(b); so the only possibility is the following: they all project in a fixed surface $S$ in $K_{3}\left(B_{i}\right)$, which is a $\gamma$-component.

Note that, by Lemma (6.8), we can suppose that $S$ project into a fixed surface $S^{\wedge}$ when we project $K_{3}\left(B_{i}\right)=K_{3}\left(\left[E^{3} / \nu_{i}\left(E^{2}\right)\right] \times E\right)$ into $K_{3}(E)$ by the natural projection on the last factor, hence $S^{\wedge}$ is $K_{3}(E)$.

Now we choose $D_{\mathrm{r}}=E_{\sigma} \times E_{s} \quad(\sigma, s$ belonging to the moduli space of elliptic curves) and generic embeddings $\nu_{i}$ in infinitely many different ways; for any choice, by using all the previous arguments, we get

-a $\gamma$-curve $C_{\sigma, s}$ in any $K_{3}\left(E_{\sigma} \times E_{s} \times E \times E\right)$,

-a surface $S_{s}$ in $K_{3}\left(E_{s} \times E \times E\right)$, ( $S_{s}$ covered by $\gamma$-curves $)$,

-a fixed surface $S$ in $K_{3}(E \times E)$ into which all $S_{s}$ project,

-a fixed surface $S^{\sim}$ in $K_{3}(E)$ into which $S$ projects, (we always use natural projections).

We want to prove that this is a contradiction to Lemma (6.6).

These facts create a situation which is very similar to a $\Delta$-situation, (see $\S 6)$ : actually, in this case, we have only a one-dimensional family of Abelian varieties: $A_{s} \times A_{s}=\left(E_{s} \times E \times E\right) \times\left(E_{s} \times E \times E\right)$ and a surface in every $A_{s} \times A_{s}$ which is the pullback, via $\pi$, of the surface $S_{s}$ contained in $K_{3}\left(E_{s} \times E \times E\right)$. So we have a fibration defined only over an open set $\Delta_{i}^{\prime} \subset \mathscr{H}_{1}$ and the surfaces $\left\{S_{s}\right\}$ are the fibres over $\Delta_{i}^{\prime}$. However, by looking at the proof of $(6.6)$, it is obvious that it is true even in this case. 
But, in our case, we also have that, if we choose $A$ isogenous to $E \times E \times E$, there are infinitely many embeddings $\mu_{p q}: E \rightarrow E^{2} \quad\left(\forall x \in E \quad \mu_{p q}(x)=(p x, q x)\right.$, $p, q$ coprime integers, the $\mu_{p q}$ are induced by the $\left.\nu_{i}\right)$ such that, when we choose a family $\left\{A_{s} \times A_{s}\right\}, s$ belonging to a suitable open set $\Delta_{p q} \subset \mathscr{H}_{1}$, (depending on $\Delta_{i}^{\prime}$ ), such that $\forall s \in \Delta_{p q}, A_{s}$ is isogenous to $E_{s} \times \mu_{p q}(E) \times E$ (as usual $A_{0}$ is isogenous to $\left[E^{2} / \mu_{p q}(E)\right] \times \mu_{p q}(E) \times E$, isogenous to $\left.A\right)$, then

$-K_{3}\left(A_{s}\right)$ contains a surface $S_{s}$ for any $s$,

$-S_{0}$ projects into a surface $S^{\prime}$ in $K_{3}(E)$ by the natural projection on the last factor, (hence condition $(*)$ is satisfied),

-all surfaces $S_{s}$ project into a fixed surface $S$ in $K_{3}\left[\mu_{p q}(E) \times E\right]$.

This is a contradiction to Lemma (6.6)!

Now we assume that $\operatorname{dim}(A)=n \geq 5$ and we proceed by induction on $n$. Suppose that for any $n$-dimensional Abelian variety $A, S^{3}(A)$, and therefore $K_{3}(A)$ contains a $\gamma$-curve; then it is true for those Abelian $n$-folds which are isogenous to $E \times B$ where $E$ is a generic elliptic curve and $B$ is a generic Abelian $(n-1)$-fold. It is easy to see that, by choosing a suitable isogeny, we also get a $\gamma$-curve in $K_{3}(B)$, and this is a contradiction to our induction hypothesis.

\section{REFERENCES}

[D-M] M. Martin-Deschamps and R. Lewin-Ménégaux, Surfaces de type général dominées par une variété fixe, Bull. Soc. Mat. France 110 (1982), 127-146.

[G] P. Griffiths, ed., Topics in transcendental algebraic geometry, Ann. of Math. Studies, no. 106, Princeton Univ. Press, 1984.

[H] R. Hartshorne, Equivalence relations on algebraic cycles and subvarieties of small codimension, Proc. Sympos. Pure Math., vol. 29, Amer. Math. Soc., Providence, R.I., 1975, pp. 129-164.

[M] D. Mumford, Rational equivalence of zero-cycles on surfaces, J. Math. Kyoto Univ. 9 (1969), 195-204.

[P] G. P. Pirola, Curves on generic Kummer varieties, Duke Math. J. 59 (1989), 73-80.

$\left[\mathbf{R}_{1}\right]$ A. A. Roitman, On $\Gamma$-equivalence of zero-dimensional cycles, Math. USSR-Sb. 15 (1971), 555-567.

[ $\left.\mathbf{R}_{2}\right] \quad$, Rational equivalence of zero-cycles, Math. USSR-Sb. 18 (1972), 571-588. ITALY

Dipartimento di Matematica, Università di Milano, via C. Saldini 50, 20133 Milano,

E-mail address: alzati@vmimat.mat.unimi.it

Dipartimento di Matematica, Università di Pavia, via Strada Nuova 65, 27100 Pavia, ITALY

E-mail address: piro23@ipvian.bitnet 